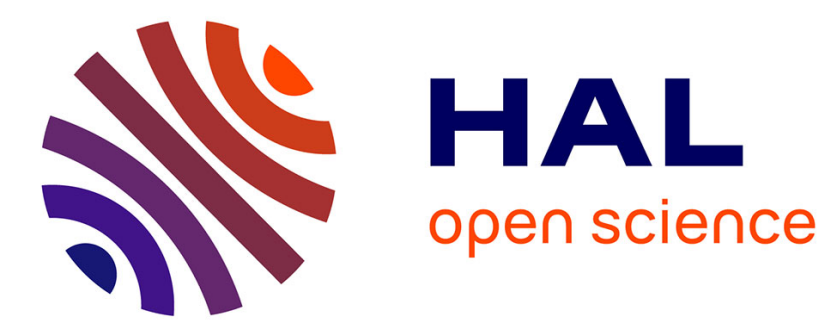

\title{
Metamagnetic behaviour of Nd3Pt23Si11
}

\author{
Christine Opagiste, Martin Jackson, Rose-Marie Galéra, Elsa Lhotel, Carley \\ Paulsen, Bachir Ouladdiaf
}

\section{To cite this version:}

Christine Opagiste, Martin Jackson, Rose-Marie Galéra, Elsa Lhotel, Carley Paulsen, et al.. Metamagnetic behaviour of Nd3Pt23Si11. Journal of Magnetism and Magnetic Materials, 2013, 340, pp.46-49. 10.1016/j.jmmm.2013.03.031 . hal-00816130

\section{HAL Id: hal-00816130 \\ https://hal.science/hal-00816130}

Submitted on 19 Apr 2013

HAL is a multi-disciplinary open access archive for the deposit and dissemination of scientific research documents, whether they are published or not. The documents may come from teaching and research institutions in France or abroad, or from public or private research centers.
L'archive ouverte pluridisciplinaire HAL, est destinée au dépôt et à la diffusion de documents scientifiques de niveau recherche, publiés ou non, émanant des établissements d'enseignement et de recherche français ou étrangers, des laboratoires publics ou privés. 


\title{
Metamagnetic behaviour of $\mathrm{Nd}_{3} \mathrm{Pt}_{23} \mathrm{Si}_{11}$
}

C. Opagiste ${ }^{\mathrm{a} *}$, M. J. Jackson ${ }^{\mathrm{a}}$, R.-M. Galéra ${ }^{\mathrm{a}}$, E. Lhotel $^{\mathrm{a}}$, C. Paulsen ${ }^{\mathrm{a}}$ and B. Ouladdiaf ${ }^{\mathrm{b}}$

anstitut Néel, CNRS-UJF, 25 avenue des Martyrs, BP 166, FR-38042 Grenoble Cédex 9, France

${ }^{b}$ Institut Laue Langevin, 6 rue Jules Horowitz, BP 156, FR-38042 Grenoble Cédex 9, France

*corresponding author: christine.opagiste@grenoble.cnrs.fr

\begin{abstract}
In the course of studying the $\mathrm{R}_{3} \mathrm{Pt}_{23} \mathrm{Si}_{11}$ series ( $\mathrm{R}=$ Rare Earth), we have recently synthetized $\mathrm{Nd}_{3} \mathrm{Pt}_{23} \mathrm{Si}_{11}$. It crystallizes in a face-centered cubic structure $(F m-3 m$ space group) with a lattice parameter of 16.8493(4) $\AA$ at room temperature. Heat capacity and magnetic measurements show the existence of a magnetic transition at $1.6 \mathrm{~K}$. Measurements of the susceptibility and magnetization down to $95 \mathrm{mK}$ reveal metamagnetic behaviour confirming antiferromagnetic ordering. Powder neutron diffraction experiments show that the magnetic propagation vector is $\mathrm{k}=[000]$ and that the 6 neodymium moments in the unit cell are antiferromagnetically coupled in pairs.
\end{abstract}

Keywords: rare earth intermetallics, metamagnetism, antiferromagnetism

\section{Introduction}

Investigation of the R-Pt-Si $(\mathrm{R}=$ rare earth element) ternary phase diagram was mainly driven by the discovery of novel ternary compounds with peculiar physical behaviour. The Ce-Pt-Si phase diagram has triggered a lot of interest [1,2] because some cerium-based compounds present interesting physical properties such as; unconventional heavy fermion superconductivity in $\mathrm{CePt}_{3} \mathrm{Si}$ [3], heavy-fermion properties in $\mathrm{CePtSi}$ [4], Kondo lattice behaviour in $\mathrm{CePtSi}_{2}$ and $\mathrm{CePt}_{2} \mathrm{Si}_{2}[5,6]$ and most recently, the observation of unusual ferromagnetic ordering in 
$\mathrm{Ce}_{3} \mathrm{Pt}_{23} \mathrm{Si}_{11}$ [7-9]. The compound $\mathrm{Yb}_{3} \mathrm{Pt}_{23} \mathrm{Si}_{11}$ has been recently synthesized and was found to be a moderately enhanced paramagnetic compound with a fairly unstable $4 \mathrm{f}$ electronic shell [10]. With the aim of finding novel compounds with remarkable magnetic properties, we have successfully synthesized new compounds in this series. In this paper, we report investigations on the magnetic behaviour of the compound $\mathrm{Nd}_{3} \mathrm{Pt}_{23} \mathrm{Si}_{11}$ at very low temperatures.

\section{Experimental Procedure}

High quality polycrystalline samples of $\mathrm{Nd}_{3} \mathrm{Pt}_{23} \mathrm{Si}_{11}$ have been prepared in an induction furnace. Stoichiometric proportions of the different constituents; Nd (99.9\%, Johnson Matthey), Pt (99.95\%, Alfa Aesar) and Si (99.9999\%, Alfa Aesar), were melted in a cold copper crucible under a highly-purified argon atmosphere. The samples were melted several times to improve the homogeneity. Mass losses during this first step were less than $0.5 \%$ and no further heat treatment was applied.

Sample structure and quality were checked by conventional X-ray powder diffraction $(\mathrm{Cu}-\mathrm{K} \alpha$ radiation on a Philips PW1730 diffractometer), whilst structural refinements were performed using the FullProf program [11]. $\mathrm{Nd}_{3} \mathrm{Pt}_{23} \mathrm{Si}_{11}$ crystallizes in the same fcc structure $(\mathrm{Fm}-3 \mathrm{~m}$ space group) as $\mathrm{Ce}_{3} \mathrm{Pt}_{23} \mathrm{Si}_{11}$ [12]. At room temperature, the value of the refined lattice parameter is 16.8493(4) A. More details on the crystallographic analysis can be found in ref [9].

Magnetization measurements were performed on an almost spherical sample of diameter $2.5 \mathrm{~mm}$ using different magnetometers based on the extraction method. In the $1.8-300 \mathrm{~K}$ temperature range, we used a commercial Quantum Design MPMS magnetometer, and a second experimental set-up, equipped with resistive detection coils and a superconducting magnet, which can supply magnetic fields of $\pm 10.5 \mathrm{~T}$. For temperatures down to $65 \mathrm{mK}$, we used a SQUID magnetometer equipped with a miniature dilution refrigerator developed at the Institut Néel.

In the $0.38-300 \mathrm{~K}$ temperature range, the heat capacity was measured using the relaxation method with a Quantum Design PPMS. 
Powder neutron diffraction experiments were carried out at the Institut Laue-Langevin (ILL) in Grenoble on the CRG (CNRS Grenoble/CSIC Spain) - two-axis diffractometer D1B using a wavelength of $2.52 \AA$, selected by a pyrolytic graphite (002). The powdered sample was installed inside a ${ }^{3} \mathrm{He}$ cryostat capable of reaching a base temperature of $400 \mathrm{mK}$.

\section{Magnetic and Thermodynamic Properties}

The inverse of the magnetic susceptibility is deduced from the isothermal magnetization curves in the temperature range $1.8-300 \mathrm{~K}$. From room temperature down to $25 \mathrm{~K}$, the inverse susceptibility varies linearly with temperature, in agreement with the Curie-Weiss law $\chi=\mathrm{C} /(T$ $\left.\theta_{p}\right)$, where $C=\mu_{\text {eff }}^{2} / 3 k_{B}$ is the theoretical Curie constant and $\mu_{e f f}=g_{J} \mu_{B}[J(J+1)]^{1 / 2}$, the effective magnetic moment of the rare earth ion. Setting the theoretical value of the effective moment of the $\mathrm{Nd}^{3+}$ ion to be $\mu_{\text {eff }}=3.62 \mu_{\mathrm{B}}$, the experimental thermal variation of the inverse susceptibility is very reproducible, adjusting the value of the paramagnetic Curie temperature $\theta_{p}$ to -15 $\pm 0.5 \mathrm{~K}$, as shown by the straight line in figure 1 . A negative value for $\theta_{p}$ suggests antiferromagnetic correlations between the rare earth ions.

Below $25 \mathrm{~K}, 1 / \chi$ deviates from the Curie-Weiss law and is characteristic of crystal electric field effects (CEF). CEF interactions partially lift the degeneracy of the fundamental multiplet $J=$ $9 / 2$, leading to a reduction of the theoretical magnetic moment, $\mu=g_{J} \mu_{\mathrm{B}} J=3.27 \mu_{\mathrm{B}}$.

Magnetization curves, in the temperature range $1.8-3 \mathrm{~K}$ are shown in figure 2 . The Arrott plots of the curves at 1.8 and $2 \mathrm{~K}$ (inset in fig. 2) intercept the $\mathrm{x}$-axis at a non-zero value, demonstrating that $\mathrm{Nd}_{3} \mathrm{Pt}_{23} \mathrm{Si}_{11}$ remains paramagnetic down to $1.8 \mathrm{~K}$. Under high fields, the magnetization tends to a maximum value of $1.5 \mu_{\mathrm{B}}$. This value is a factor of two smaller than the theoretical saturated moment $3.27 \mu_{\mathrm{B}}$, strongly suggesting the reduction of the magnetic moment by the CEF interactions. 


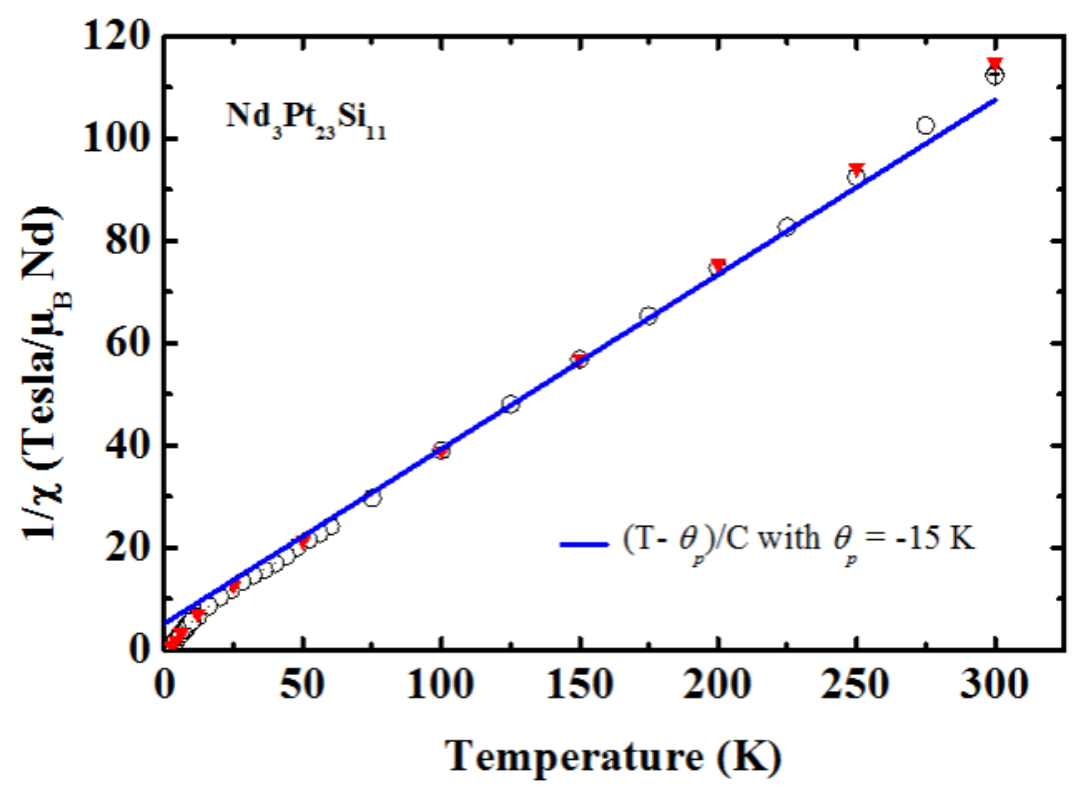

Fig. 1: Thermal variation of the inverse susceptibility of $\mathrm{Nd}_{3} P t_{23} S i_{11}$. The open black dots represent the susceptibility deduced from measurements in magnetic fields up to 8 Tesla using the in-house magnetometer with resistive detection coils. The full red triangles represent the susceptibility deduced from the measurements using the commercial Quantum Design MPMS magnetometer. The straight line corresponds to the Curie-Weiss law calculated with the theoretical values of the effective moment of the $\mathrm{Nd}^{3+}$ ion.

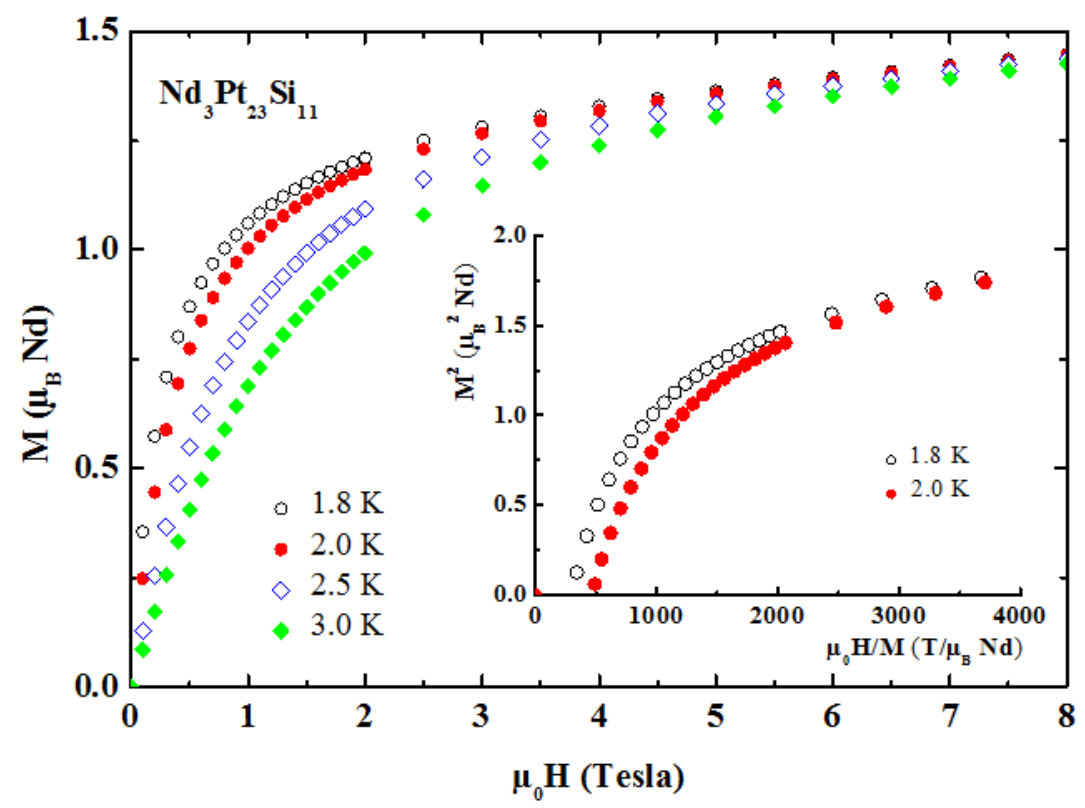

Fig. 2: The field dependence of the magnetization between 1.8 and $3 \mathrm{~K}$. The inset shows the Arott's plots of the magnetization at 1.8 and $2 \mathrm{~K}$. 
Specific heat measurements, displayed in figure 3, show that a transition occurs at $1.6 \mathrm{~K}$. The steep initial slope of the magnetization at $1.8 \mathrm{~K}$ points to ferromagnetic-type behaviour, in an apparent contradiction with the negative value found for $\theta_{p}$. The entropy associated with the lambda anomaly of the specific heat is close to $\mathrm{R} \ln (2)$, thus confirming a doublet CEF ground state for the $\mathrm{Nd}^{3+}$ ions. In order to gain further insight as to the nature of this transition, magnetic susceptibility measurements were performed down to $95 \mathrm{mK}$.

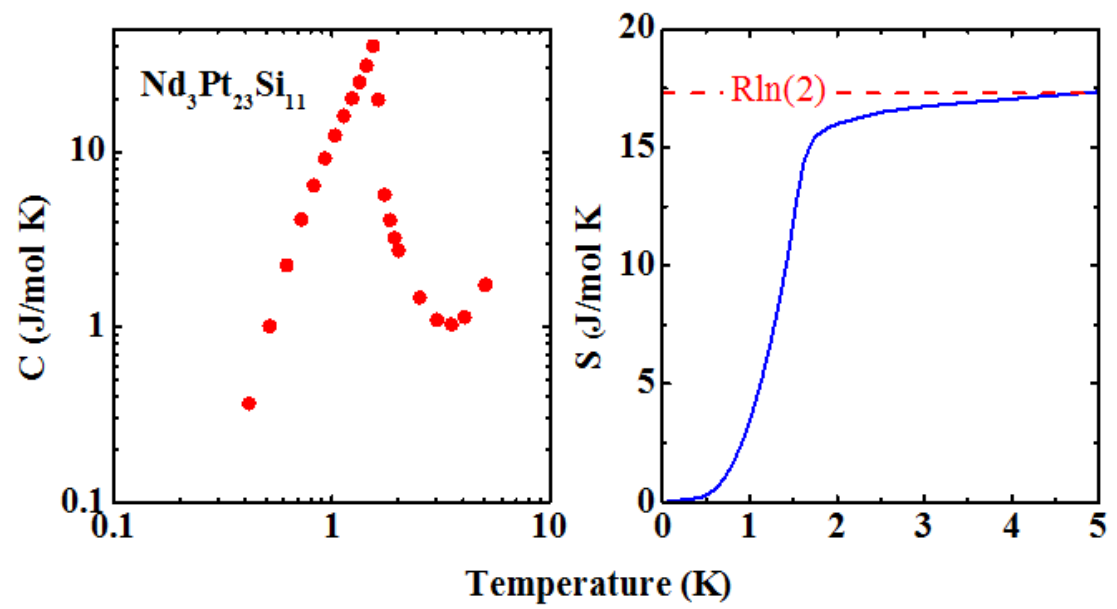

Fig.3: Left: thermal variation of the heat capacity in $\mathrm{Nd}_{3} P t_{23} \mathrm{Si}_{11}$. Right: entropy associated with the lambda anomaly in the specific heat.

As shown in figure 4 (a), one observes a sharp peak at $T^{*}=1.6 \mathrm{~K}$ in the susceptibility, which is characteristic of a magnetic phase transition. The value of $T^{*}$ is in very good agreement with the transition temperature observed in the heat capacity measurements. At first glance, this transition looks ferromagnetic because of the steep increase of $\chi$ when $T$ tends to $1.6 \mathrm{~K}$ in the paramagnetic phase, and the positive intercept of $1 / \chi$ with the temperature axis $\theta_{p}^{L T}=+1.5 \mathrm{~K}$ (see fig. 4 (b)). However, the maximum value of $\chi=0.035 \mathrm{emu} / \mathrm{cm}^{3}$ is one order of magnitude less than the expected value at $T_{C}$ for a ferromagnet, $\chi_{\max }=1 / N$, where $N$ is the demagnetizing factor. For a spherical sample, which is almost the case here, $\mathrm{N}=3$, leading to $\chi_{\max }=1 / 3$ $\mathrm{emu} / \mathrm{cm}^{3}$.

The magnetization curves measured at $95 \mathrm{mK}$ and $1.8 \mathrm{~K}$ using the set-up with the ${ }^{3} \mathrm{He}$ dilution 
in fields up to $8 \mathrm{~T}$ are shown in figure 5 (a) and compared with the one measured at $1.8 \mathrm{~K}$ using the set-up with the ${ }^{4} \mathrm{He}$ cryostat. It can be seen that the magnetization curves at $1.8 \mathrm{~K}$ are in full agreement. Above $4 \mathrm{~T}$, the curves at $95 \mathrm{mK}$ and $1.8 \mathrm{~K}$ superpose and reach the same value of $1.45 \mu_{\mathrm{B}}$ at $8 \mathrm{~T}$. The Arrott plot at $95 \mathrm{mK}$ (inset fig. 5 (a)) is consistent with antiferromagnetic behaviour. Figure 5 (b) shows the magnetization loop measured in the low field region at $95 \mathrm{mK}$. At $0.1 \mathrm{~T}$, a magnetization jump is observed. In the loop at $1.3 \mathrm{~K}$ (not shown here) a similar jump is observed at $0.08 \mathrm{~T}$, highlighting the metamagnetic behaviour in the ordered phase. A non-negligible hysteresis is associated to the metamagnetic transition. The fact that this transition occurs under very low fields suggests a weak magnetic anisotropy. We also observe a very small remanent magnetization of $\pm 0.02 \mu_{\mathrm{B}}$, which could be reasonably ascribed to a small amount of ferromagnetic impurities. All of this leads us to the conclusion that the transition at $\mathrm{T}^{*}$ is antiferromagnetic.

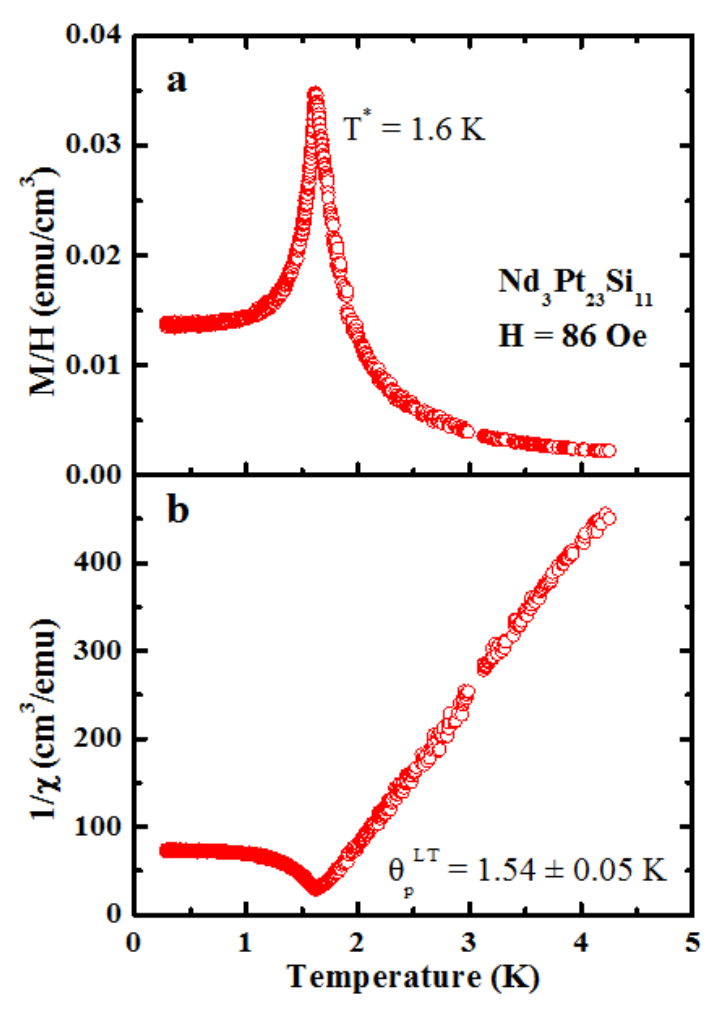

Fig. 4: (a) DC susceptibility M/H as a function of temperature measured with an applied field of 86 Oe. (b) DC inverse susceptibility $1 / \chi=H / M$. The extrapolation of the linear part of the inverse susceptibility below $4 \mathrm{~K}$ yields a positive value for $\theta_{p}, \theta_{p}^{L T}=1.5 \mathrm{~K}$. 

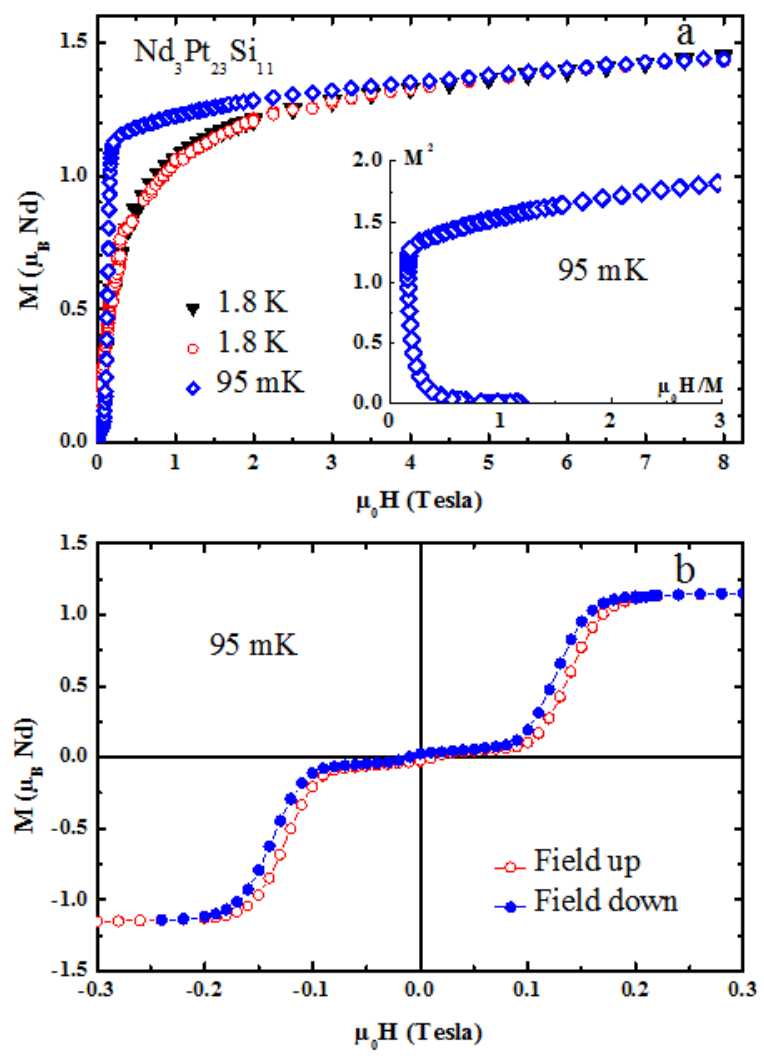

Fig. 5: (a) Magnetization curves measured below and above $1.6 \mathrm{~K}$ in fields up to $8 \mathrm{~T}$. The open symbols are the data obtained using the ${ }^{3}$ He dilution refrigerator whereas the closed symbols were obtained using $a^{4}$ He cryostat The inset shows the Arrott plot of the magnetization curve at $95 \mathrm{mK}$. (b) Magnetization loop in low fields at $95 \mathrm{mK}$.

\section{Powder Neutron Diffraction Studies}

In order to check the magnetic arrangement in the ordered phase, powder neutron diffraction studies have been performed down to $400 \mathrm{mK}$. To ensure good thermal coupling at these very low temperatures, it is necessary to use a sealed copper sample holder with a low He pressure. Therefore, in the collected diffraction patterns, two very intense peaks due to the copper diffraction are present at $2 \theta=74.49$ and 88.69 degrees. Due to the Q-dependence of the magnetic form factor of the rare earth ions, the stronger magnetic signal is observed in the low $2 \theta$ region. Also, since the $\mathrm{CEF}$ effects reduce the magnetic moment of the $\mathrm{Nd}$ ions, one expects a small magnetic contribution to the powder diffraction pattern. This makes the powder data analysis rather difficult. 


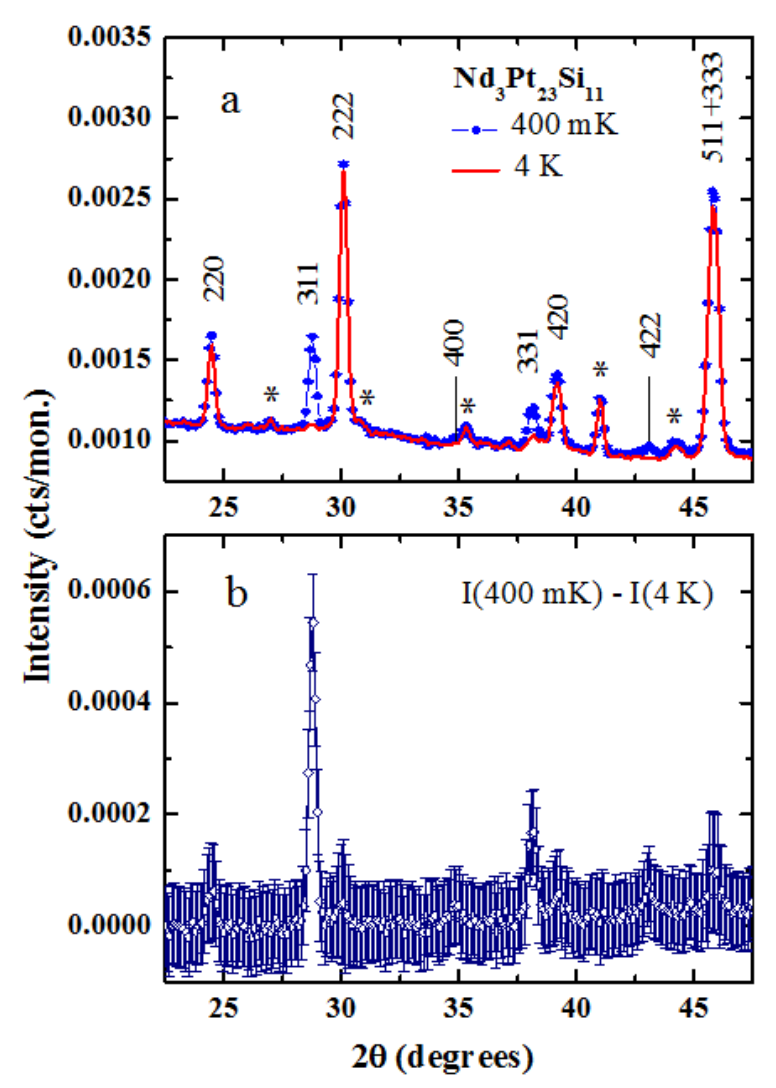

Fig. 6: (a) Comparison between the powder patterns at $400 \mathrm{mK}$ and $4 \mathrm{~K}$ in $\mathrm{Nd}_{3} \mathrm{Pt}_{23} \mathrm{Si}_{11}$. The stars mark the peaks due to impurity phases. (b) Pattern difference between $400 \mathrm{mK}$ and $4 \mathrm{~K}$.

In figure 6 (a), we compare the diffraction patterns collected at $400 \mathrm{mK}$ and $4 \mathrm{~K}$. In both patterns, some peaks cannot be indexed in the crystallographic structure and we attribute them to impurity phases. At $400 \mathrm{mK}$, we observe no extra peaks at the positions forbidden by the crystallographic structure. Thus, as in $\mathrm{Ce}_{3} \mathrm{Pt}_{23} \mathrm{Si}_{11}$ [8], the fcc lattice translations are also translations for the magnetic structure in $\mathrm{Nd}_{3} \mathrm{Pt}_{23} \mathrm{Si}_{11}$ and the magnetic propagation vector is then $\mathrm{k}=[000]$. Only the intensity of the (311) and (331) reflections increases significantly in the ordered phase, as illustrated in figure 6 (a). The pattern difference (fig. 6 (b)), reveals a tiny intensity for the peaks with even Miller indices, however the intensity is less than the experimental uncertainty. Figure 7 clearly shows that the intensity at the (311) position increases while the temperature is lowered, confirming a magnetic contribution. However, the thermal evolution of the (222) reflection is less obvious. 


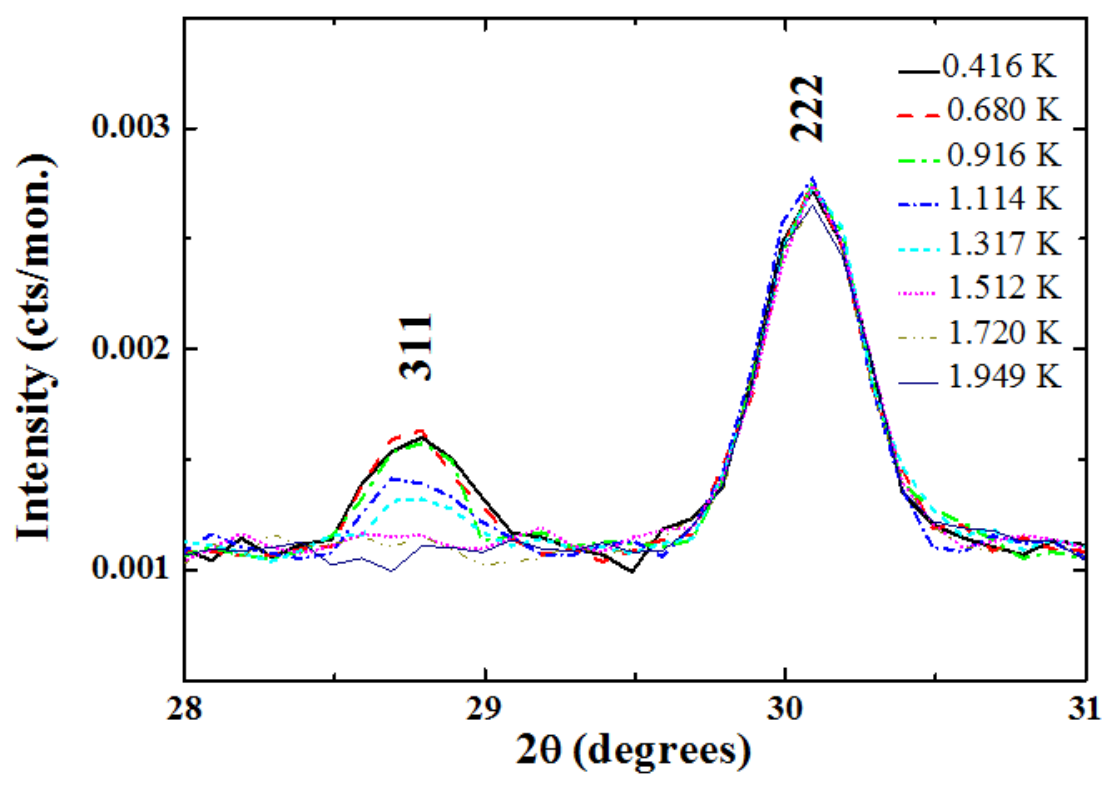

Fig. 7: Thermal variation of the intensity at the positions (311) and (222).

The Nd ions occupy the $24 d$ sites of the $F m-3 m$ space group. They are located at the positions: $\mathrm{Nd}_{1}(0.25,0.25,0), \mathrm{Nd}_{2}(0.25,-0.25,0), \mathrm{Nd}_{3}(0,0.25,0.25), \mathrm{Nd}_{4}(0,0.25,-0.25), \mathrm{Nd}_{5}(0.25,0,0.25)$ and $\mathrm{Nd}_{6}(-0.25,0,0.25)$. Group theory analysis was used to determine the magnetic configurations of $\mathrm{Nd}$ atoms in the $F m-3 m$ space group. The propagation vector being $\mathrm{k}=[000]$, the relevant irreducible representations (IR) of the magnetic structure are those of the point group $m-3 m$. There are ten irreducible representations labelled $\Gamma_{1}, \Gamma_{2}, \Gamma_{3}, \Gamma_{4}$, of dimension $1, \Gamma_{5}, \Gamma_{6}$, of dimension $2, \Gamma_{7}, \Gamma_{8}, \Gamma_{9}$ and $\Gamma_{10}$ of dimension 3 . The reduction of the magnetic or induction representation of the site $24 d$ gives $\Gamma_{2 d d}=\Gamma_{3}+\Gamma_{6}+2 \Gamma_{8}+3 \Gamma_{10}$. The basis vectors, which span the space of a given irreducible representation, are obtained by the projection operator technique $[13,14]$. The only non-zero basis vectors of the site $24 d$ are those of $\Gamma_{3}, \Gamma_{6}, \Gamma_{8}$ and $\Gamma_{10}$. The basis vectors of $\Gamma_{6}$ and $\Gamma_{8}$ describe complex non collinear antiferromagnetic arrangements, while those of $\Gamma_{10}$ correspond to a ferromagnetic one. However the one dimensional irreducible representation $\Gamma_{3}$ describes a simple antiferromagnetic non collinear configuration along the principal axes [001], [100] and [010] for $\mathrm{Nd}_{1}$ and $\mathrm{Nd}_{2}, \mathrm{Nd}_{3}$ and $\mathrm{Nd}_{4}$, and $\mathrm{Nd}_{5}$ and $\mathrm{Nd}_{6}$ respectively. The corresponding magnetic Shubnikov group is $F m^{\prime}-3^{\prime} m$. 


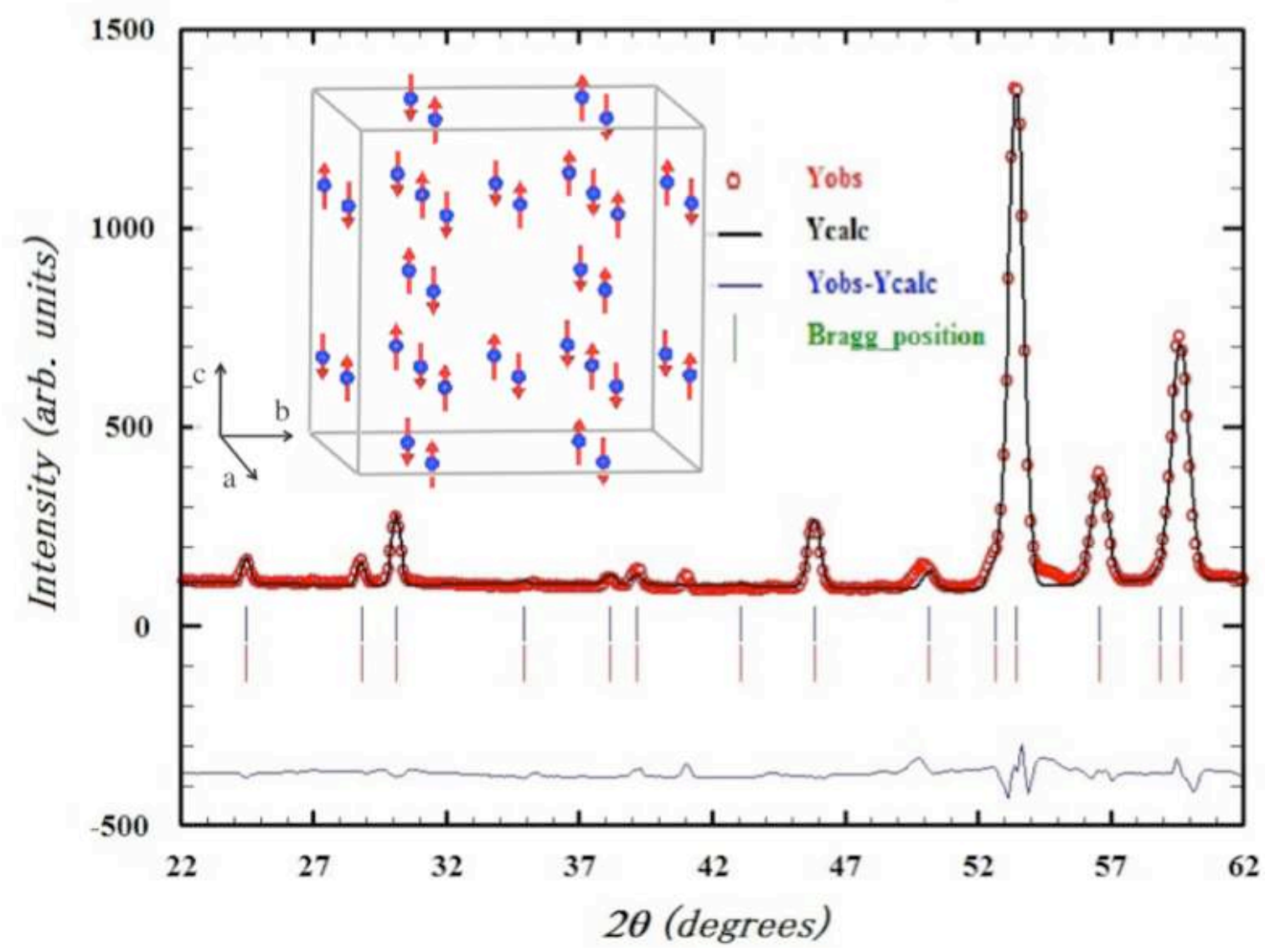

Fig. 8: Rietveld refinement of the neutron diffraction pattern at $400 \mathrm{mK}$. The magnetic structure is accounted for by an antiferromagnetic coupling between the magnetic moments at $N d_{1}$ $(0.25,0.25,0)$ and $N d_{2}(0.25,-0.25,0), N d_{3}(0,0.25,0.25)$ and $N d_{4}(0,0.25,-0.25), N d_{5}(0.25,0,0.25)$ and $N d_{6}(-0.25,0,0.25)$, respectively (see inset). The value of the magnetic moment refined at 400 $m K$ is $1.45 \pm 0.16 \mu_{B} / N d$.

The refinements of the powder patterns at $4 \mathrm{~K}$ and $400 \mathrm{mK}$, were performed using the FullProf program [11]. At both temperatures the refined lattice parameter is a $=16.8097(7) \AA$ and the refined values of the atomic coordinates are found in very good agreement with those obtained at room temperature [9]. The model of magnetic structure used in the refinements of the powder pattern corresponds to an antiferromagnetic coupling between the magnetic moments at $\mathrm{Nd}_{1}$ and $\mathrm{Nd}_{2}, \mathrm{Nd}_{3}$ and $\mathrm{Nd}_{4}, \mathrm{Nd}_{5}$ and $\mathrm{Nd}_{6}$, respectively, with the moments along the [001] axis (see fig. 8). At $400 \mathrm{mK}$, the refined value of the moment that gives the best agreement between the observed and calculated intensities for the (311) and (331) reflections is $1.45 \pm 0.16 \mu_{\mathrm{B}} / \mathrm{Nd}$. The reliability 
factors for the nuclear and the magnetic refinements and the value of $\chi^{2}$ are 6.52, 26.2 and 2.02 respectively. The refined value of the moment is also in good agreement with the bulk magnetization results. Note that conversely, in the $\mathrm{Ce}_{3} \mathrm{Pt}_{23} \mathrm{Si}_{11}$ compound, where the Ce ions occupy the same $24 d$ sites, a ferromagnetic coupling was found between the magnetic moments at $\mathrm{Ce}_{1}$ and $\mathrm{Ce}_{2}, \mathrm{Ce}_{3}$ and $\mathrm{Ce}_{4}, \mathrm{Ce}_{5}$ and $\mathrm{Ce}_{6}$, respectively ( $\left.\Gamma_{10} \mathrm{IR}\right)$.

\section{Conclusion}

Our study of the magnetic and thermodynamic properties of the new compound $\mathrm{Nd}_{3} \mathrm{Pt}_{23} \mathrm{Si}_{11}$ reveals that it presents an antiferromagnetic phase transition at $\mathrm{T}_{\mathrm{N}}=1.6 \mathrm{~K}$. Magnetization and specific heat results strongly suggest that the CEF ground state of the $\mathrm{Nd}$ ions is a doublet. In the antiferromagnetic phase, the magnetization reveals metamagnetic behaviour. However, the low value of the critical field indicates that the magnetic anisotropy is small. Preliminary studies of the antiferromagnetic phase by powder neutron diffraction show that the magnetic structure has the same periodicity as the crystallographic one, $\mathrm{k}=[000]$. The value of the magnetic moment refined at $400 \mathrm{mK}$ is $1.45 \pm 0.16 \mu_{\mathrm{B}} / \mathrm{Nd}$. Further neutron diffraction studies on single crystals in applied magnetic fields are required to determine the actual magnetic arrangement in the antiferromagnetic phase.

\section{Acknowledgments}

We are grateful to A. Hadj-Azzem, J. Balay, D. Dufeu, E. Eyraud and P. Lachkar from the Institut Néel for their technical assistance. We are pleased to thank V. Nassif and all the staff of D1B CRG, together with O. Losserand of ILL SANE for their valuable help during the experiments on D1B. 


\section{References}

[1] A. Gribanov, Y. D. Seropegin, A.I. Tursina, O. I. Bodak, P. Rogl, H. Noel, J. Alloys Compd. 383, 286 (2004).

[2] A. Gribanov, A. Grytsiv, Y. Seropegin, E. Royanian, P. Rogl, E. Bauer, G. Giester, Y. Seropegin, J. Solid State Chem. 181, 2964 (2008).

[3] E. Bauer, H. Kaldarar, A. Prokofiev, E. Royanian, A. Amato, J. Sereni, W. BramerEscamilla, I. Bonalde, J. Phys. Soc. Jpn. 76 (5) 051009 (2007).

[4] W.H. Lee, H.C. Ku, R.N. Shelton, Phys. Rev. B 38, 11562 (1988).

[5 ] W.H. Lee, K.S. Kwan, P. Klavins, R.N. Shelton, Phys. Rev. B 42, 6542 (1990).

[6] B.H. Grier, J.M. Lawrence, S. Horn, J.D. Thompson, J. Phys. C: Solid State Phys. 21, 1099 (1988).

[7] C. Opagiste, C. Paulsen, E. Lhotel, P. Rodière, R.-M. Galéra, P. Bordet, and P. Lejay, J. Magn. Magn. Mater. 321, 613 (2009).

[8] C. Opagiste, R.-M. Galéra, M. Amara, C. Paulsen, S. Rols, B. Ouladdiaf, Phys. Rev. B 84, $134401(2011)$

[9] C. Opagiste, R.-M. Galéra, J. Alloys Compd. 541, 403 (2012).

[10] D. Kaczorowski, A. Gribanov, S. Safronov, P. Rogl, Y.Seropegin, J. Alloys Compd. 509, 8987-8990 (2011).

[11] J. Rodriguez-Carvajal, Satellite Meeting on Powder Diffraction of the XV Congress of the IUCr, Book of Abstracts, Toulouse, France, 1990, p. 127.

[12] A. I. Tursina, A. V. Gribanov, Y. D. Seropegin, K. V. Kuyukov, and O. I. Bodak, J. Alloys Compd. 347, 121 (2002).

[13] G. Y. Lyubarskii, The Application of Group Theory in Physics (Pergamon, Oxford, 1960). [14] V. Heine, Group Theory in Quantum Mechanics (Pergamon, Oxford, 1960), Appendix K. 